\title{
Novel Method for Detection of Reactive Oxygen Species In Vivo in Human Skeletal Muscle
}

\author{
J. D. LA FAVOR ${ }^{1,4}$, E. J. ANDERSON ${ }^{2,4}$, R. C. HICKNER ${ }^{1,3,4,5,6}$ \\ ${ }^{1}$ Department of Kinesiology, Human Performance Laboratory, East Carolina University, Greenville, \\ NC, USA, ${ }^{2}$ Department of Pharmacology and Toxicology, East Carolina University, Greenville, \\ NC, USA, ${ }^{3}$ Department of Physiology, East Carolina University, Greenville, NC, USA, ${ }^{4}$ East \\ Carolina Diabetes and Obesity Institute, East Carolina University, Greenville, NC, USA, ${ }^{5}$ Center \\ for Health Disparities, East Carolina University, Greenville, NC, USA, ${ }^{6}$ Department of Biokinetics, \\ Exercise and Leisure Sciences, University of KwaZulu-Natal, Durban, South Africa
}

Received June 7, 2013

Accepted October 17, 2013

On-line February 24, 2014

\begin{abstract}
Summary
Excessive production of reactive oxygen species (ROS) are implicated in the pathogenesis of numerous disease states. However, direct measurement of in vivo ROS in humans has remained elusive due to limited access to appropriate tissue beds and the inherently short half-lives and high reactivity of ROS. Herein, we describe a novel technique by which to measure in vivo ROS in human skeletal muscle. Microdialysis probes were inserted into the vastus lateralis of eight healthy volunteers. Amplex Ultrared, a highly specific fluorogenic substrate for hydrogen peroxide $\left(\mathrm{H}_{2} \mathrm{O}_{2}\right)$, and horseradish peroxidase (HRP), were perfused through microdialysis probes, and outflowing dialysate was collected and fluorescence was measured. Extracellular $\mathrm{H}_{2} \mathrm{O}_{2}$ that crossed the microdialysis membrane was measured via fluorescence of the dialysate. Superoxide dismutase (SOD) was then added to the inflowing perfusion media to convert any superoxide crossing the microdialysis membrane to $\mathrm{H}_{2} \mathrm{O}_{2}$ within the microdialysis probe. Fluorescence significantly increased $(\mathrm{P}=0.005)$ upon SOD addition. These data demonstrate the feasibility of measuring both in vivo $\mathrm{H}_{2} \mathrm{O}_{2}$ and superoxide in the extracellular environment of human skeletal muscle, providing a technique with a potential application to a wide range of circulatory and metabolic studies of oxidative stress.
\end{abstract}

\section{Key words}

Superoxide • Hydrogen peroxide • Microdialysis • Amplex red • Oxidative stress

\section{Corresponding author}

Justin D. La Favor, Johns Hopkins University School of Medicine, Department of Urology, 600 N. Wolfe St., Marburg 414, Baltimore, MD 21287, USA. Fax: +1 (410) 614-3695. E-mail: jlafavo3@jhmi.edu

Oxidative stress is traditionally used to describe situations in which the generation of reactive oxygen species (ROS) overwhelms antioxidant defense systems, resulting in oxidative damage to tissue and macromolecules. Superoxide $\left(\mathrm{O}_{2}{ }^{--}\right)$is considered the parent ROS molecule, which is a highly reactive molecule that can be dismutated either enzymatically by superoxide dismutase (SOD) into hydrogen peroxide $\left(\mathrm{H}_{2} \mathrm{O}_{2}\right)$, or spontaneously into $\mathrm{H}_{2} \mathrm{O}_{2}$, peroxynitrite $\left(\mathrm{ONOO}^{-}\right)$, or the hydroperoxyl radical $\left(\mathrm{HOO}^{-}\right)$(Murrant and Reid 2001). ROS act as intracellular signaling molecules that modulate many biological processes (Poli et al. 2004); however, in the state of excessive ROS levels the signaling process acts to cause damage to lipids, proteins, and DNA. As a consequence, apoptosis and increased cellular permeability may occur, which 
lead to inflammation, endothelial dysfunction, and vascular remodeling, all of which contribute to cardiovascular and metabolic disease pathogenesis (Weseler and Bast 2010).

Oxidative stress has been implicated in the pathogenesis of several diseases and detrimental processes, such as hypertension, atherosclerosis, heart failure, diabetes mellitus, neurodegeneration, cancer, and aging (Dikalov 2011). Despite the important physiological and pathological roles of ROS, assessment of oxidative stress in humans often relies on measurement of indirect byproducts of lipid peroxidation in serum or plasma (Janero 1990). Direct measurement of in vivo ROS levels in humans has remained elusive due to limited access to appropriate tissue beds and the inherently short half-lives and high reactivity of ROS. In the present study, we describe a novel method in which to measure in vivo $\mathrm{H}_{2} \mathrm{O}_{2}$ and $\mathrm{O}_{2}{ }^{--}$production in human skeletal muscle interstitial space. Importantly, $\mathrm{H}_{2} \mathrm{O}_{2}$ and $\mathrm{O}_{2}{ }^{-}$appear to be the predominant ROS molecules in skeletal muscle (Fisher-Wellman and Neufer 2012).

Young (age 21-29), healthy men were recruited for participation in this study. All subjects were nonsmokers with no known history of cardiovascular disease, and were not taking medications for hypertension, hypercholesterolemia, or insulin resistance. All subjects abstained from antioxidant supplementation for at least two weeks prior to testing. All subjects provided written informed consent, and all procedures were approved by the University and Medical Center Institutional Review Board of East Carolina University.

A microdialysis probe was inserted under sterile technique into the left vastus lateralis of each subject as previously described (Hickner et al. 1994), while the subject was resting in a hospital bed. Following administration of local anesthesia ( $1 \mathrm{ml}$ of $1 \%$ Lidocaine $\mathrm{HCl}$ ) above the muscle fascia, an 18-gauge catheter (Jelco, Smiths Medical, Southington, CT) surrounded by plastic introducer tubing (CMA Microdialysis AB, Solna, Sweden) was inserted into the vastus lateralis. The catheter was withdrawn, while the introducer tubing was left in the muscle. A microdialysis probe (CMA 20 Elite, CMA Microdialysis) was inserted into the introducer, and the splittable tubing was pulled out of the thigh, leaving the probes in place in the muscle bed.

Probes were perfused with a $0.9 \%$ saline solution with a microinfusion pump (CMA 107, CMA
Microdialysis) at a flow rate of $2.0 \mu \mathrm{l} / \mathrm{min}$ for the remainder of the experiment. The distal $10 \mathrm{~mm}$ of the microdialysis probe contains a semi-permeable membrane allowing for bi-directional diffusion of small molecules $(<20 \mathrm{kDa})$. Probes were perfused for $90 \mathrm{~min}$ to allow for recovery from trauma induced by probe insertion. Amplex Ultrared ( $100 \mu \mathrm{M}$ final concentration; Molecular Probes, Eugene, OR) and horseradish peroxidase (HRP; $1.0 \mathrm{U} / \mathrm{ml}$ final concentration; Sigma Aldrich, St. Louis, MO) were added to the perfusate. Amplex Ultrared is a fluorogenic substrate with a very low background fluorescence which, in the presence of HRP, reacts with $\mathrm{H}_{2} \mathrm{O}_{2}$ with a $1: 1$ stoichiometry to produce the highly fluorescent resorufin (Mohanty et al. 1997, Zhou et al. 1997). Perfusion of these substrates allows $\mathrm{H}_{2} \mathrm{O}_{2}$ in the muscle extracellular environment to cross over the membrane to produce the fluorescent resorufin within the microdialysis probe. The molecular size of HRP is much greater than the pore size of the microdialysis probes, ensuring that all resorufin production takes place within the microdialysis probe. Three 20 min dialysate (probe outflow) samples were collected in $150 \mu \mathrm{l}$ polyethylene collection vials and analyzed immediately upon collection. $30 \mu \mathrm{l}$ of dialysate was added to a $250 \mu \mathrm{l}$ borosilicate cuvette (Wheaton Industries, Millville, NJ) with a gel-loading pipet tip, and fluorescence intensity was measured with a TD-700 laboratory fluorometer (Turner Designs, Sunnyvale, CA) fit with a minicell adaptor kit (Turner Designs), at an excitation wavelength of $550 \mathrm{~nm}$ and an emission wavelength of $570 \mathrm{~nm}$. A platform was inserted into the minicell to raise the cuvette position such that $30 \mu \mathrm{l}$ of sample covered the optical window of the fluorometer. SOD $(10 \mathrm{U} / \mathrm{ml}$ final concentration; Sigma Aldrich) was then added to the perfusate allowing for the conversion of $\mathrm{O}_{2}{ }^{--}$that crosses over the membrane to $\mathrm{H}_{2} \mathrm{O}_{2}$, which reacts with the fluorogenic substrate. Like HRP, the molecular size of SOD is much greater than the pore size of the microdialysis probes, ensuring that all conversion of $\mathrm{O}_{2}{ }^{--}$to $\mathrm{H}_{2} \mathrm{O}_{2}$ occurs within the microdialysis probe. Three 20 min dialysate samples were collected and fluorescence intensity was measured. The infusion pump and all collection vials were wrapped in aluminum foil throughout the duration of the experiment to prevent room light-mediated photochemical oxidation of Amplex Ultrared at any time or site throughout experimentation. 

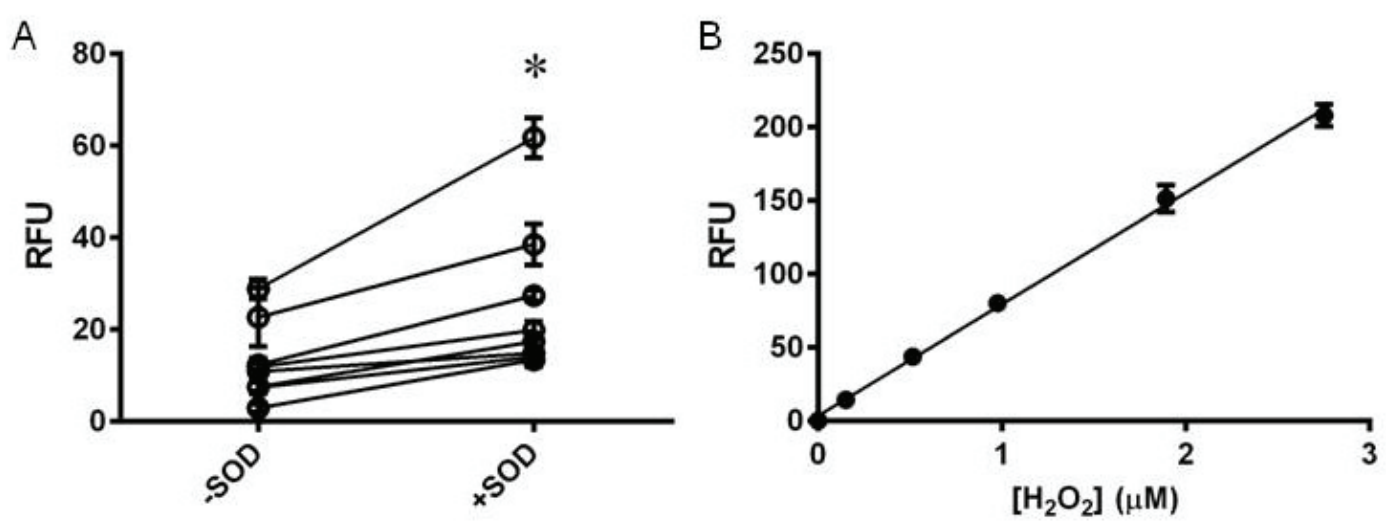

Fig. 1. (A) Fluorometric analysis of in vivo reactive oxygen species (ROS). Hydrogen peroxide $\left(\mathrm{H}_{2} \mathrm{O}_{2}\right)$ was measured in the skeletal muscle of $n=8$ individuals in the absence (-SOD) and presence (+SOD) of superoxide dismutase (SOD) in the perfusate. Background fluorescence of the perfusate ranged from 1-3 relative fluorescence units (RFU), and was subtracted from fluorescence of the dialysate, expressed in RFU. -SOD values strictly reflect $\mathrm{H}_{2} \mathrm{O}_{2}$ that diffuses into the probe, while the increased fluorescence upon SOD addition reflects the additional contribution by superoxide. * Fluorescence was increased upon addition of SOD to the perfusate $(P=0.005)$. Connecting lines indicate results from the same individual. Values are expressed as mean \pm SEM of three replicates of each measurement. (B) $\mathrm{H}_{2} \mathrm{O}_{2}$ standard curve. Perfusates were subjected to varying concentrations of $\mathrm{H}_{2} \mathrm{O}_{2}$ prepared from a stock solution. The background fluorescence of the perfusate was used as the zero standard and subtracted off of the fluorescence of the $\mathrm{H}_{2} \mathrm{O}_{2}$ standards. Values are means \pm SEM for $n=8$ trials.

Fluorescence of all dialysate samples were above the detection limit of the fluorometer (Fig. 1A), demonstrating the utility of the Amplex Ultrared fluorescent probe for use in this manner. A paired t-test reveals that fluorescence intensity was significantly elevated when SOD was added to the perfusate, indicating that $\mathrm{O}_{2}{ }^{-}$is capable of crossing the microdialysis membrane. An $\mathrm{H}_{2} \mathrm{O}_{2}$ standard curve was conducted following each experiment by addition of known concentrations of $\mathrm{H}_{2} \mathrm{O}_{2}$ to $30 \mu \mathrm{l}$ aliquots of the remaining perfusate, allowing for conversion of relative fluorescence units to $\left[\mathrm{H}_{2} \mathrm{O}_{2}\right]$. The $\mathrm{H}_{2} \mathrm{O}_{2}$ standard curve (Fig. 1B) demonstrates the linearity of resorufin production in response to $\mathrm{H}_{2} \mathrm{O}_{2}$, and demonstrates the ability to accurately measure $\mathrm{H}_{2} \mathrm{O}_{2}$ in the low micromolar range with the concentrations of Amplex Ultrared and HRP utilized in this study. This standard curve indicates that the $\left[\mathrm{H}_{2} \mathrm{O}_{2}\right]$ detected by this system in these subjects is $\sim 0.16 \mu \mathrm{M}$ without addition of SOD, and $\sim 0.32 \mu \mathrm{M}$ with the addition of SOD. It should be acknowledged that recovery of $\mathrm{H}_{2} \mathrm{O}_{2}$ from the interstitial fluid is likely to be less than $100 \%$ at the perfusion flow rate and membrane size used, indicating that the $\left[\mathrm{H}_{2} \mathrm{O}_{2}\right]$ determined by this method is not equal to the absolute interstitial $\left[\mathrm{H}_{2} \mathrm{O}_{2}\right]$. Preliminary studies were performed with SOD in the perfusate, but without Amplex Ultrared or HRP added to the perfusate. In these preliminary studies, dialysate was added directly to a saline solution containing HRP and Amplex Ultrared, from which fluorescence was measured. All of these experiments failed to produce a fluorescence reading above the detection limit, suggesting that $\mathrm{H}_{2} \mathrm{O}_{2}$ may diffuse out of the membrane after entering the membrane, or that $\mathrm{H}_{2} \mathrm{O}_{2}$ may spontaneously decompose into $\mathrm{H}_{2} \mathrm{O}$ during the 20 min collection period. These preliminary studies demonstrate the necessity of addition of Amplex Ultrared and HRP to the perfusate to form resorufin rapidly upon $\mathrm{H}_{2} \mathrm{O}_{2}$ diffusion into the membrane.

It has previously been reported that reduced glutathione (GSH) and $\beta$-nicotinamide adenine dinucleotide (NADH) may induce auto-oxidation of the Amplex Red/HRP assay (Votyakova and Reynolds 2004). Because both GSH and NADH are small enough to cross over the microdialysis membrane, we performed in vitro experiments to test their auto-oxidative capacity in the microdialysis system. Microdialysis probes were perfused as described above, with the microdialysis membrane submerged in a beaker containing varying concentrations of GSH (Sigma Aldrich) or NADH (Agros Organics) mixed in saline, and fluorescence of the dialysate was measured. GSH concentrations of $0.5,1.0$, 5.0 , and $10 \mathrm{mM}$ were tested, which did not induce any detectable fluorescence (data not shown). NADH concentrations below $10 \mu \mathrm{M}$ did not induce an appreciable amount of auto-oxidation (Fig. 2). The cellular NADH concentration has been estimated at $110 \mathrm{nM}$ (Zhang et al. 2002), thus physiological NADH is unlikely to influence ROS detection with this method. However, supraphysiological concentrations of NADH did induce apparent auto-oxidation, which was partially 
prevented by addition of SOD to the perfusate (Fig. 2). These results suggest that it is unlikely that Amplex Ultrared is auto-oxidized by GSH or NADH in an in vivo setting, but indicate that the methods described herein are not suitable to detect ROS in a system where exogenous NADH is added.

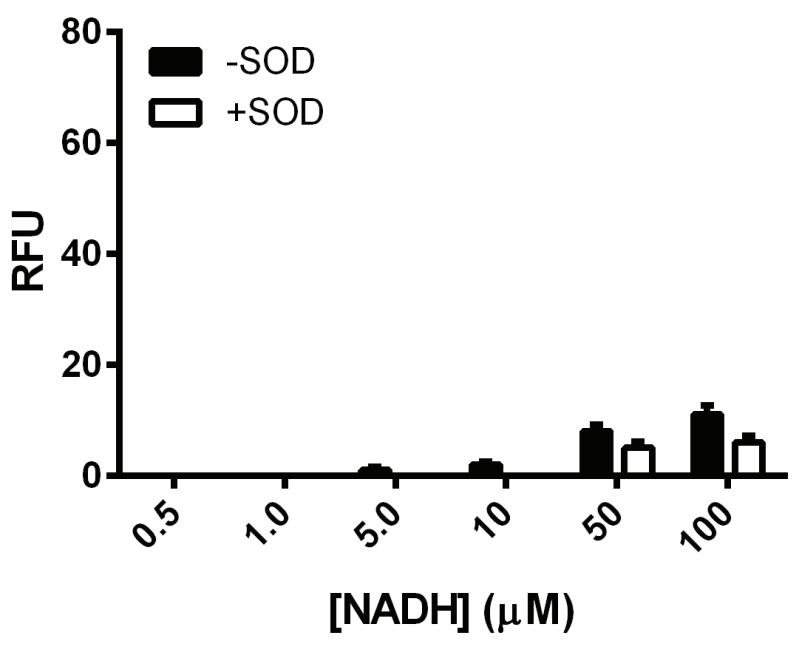

Fig. 2. Auto-oxidation of Amplex Ultrared by in vitro exposure to $\mathrm{NADH}$. Microdialysis probes were perfused with the Amplex Ultrared/HRP mixture, in the absence (-SOD) and presence (+SOD) of superoxide dismutase (SOD) in the perfusate. The microdialysis membrane was submerged in a beaker containing the indicated concentration of $\mathrm{NADH}$, and fluorescence of dialysate measured. The background fluorescence of the perfusate of 2 relative fluorescence units (RFU) was subtracted from the fluorescence of each sample. Values are expressed as mean \pm SEM of three replicates of each measurement.

Microdialysis has previously been utilized to measure extracellular $\mathrm{O}_{2}{ }^{--}$in rodent (Close et al. 2007, McArdle et al. 2001, 2004, Pattwell et al. 2001) and human (Hellsten et al. 2007) skeletal muscle, particularly in response to electrically stimulated muscle contractions or exercise. These investigations have utilized cytochrome $\mathrm{c}$ reduction for $\mathrm{O}_{2}{ }^{--}$detection. We believe that the Amplex Ultrared technique described in the present study affords several advantages over the cytochrome c reduction assay. Particularly, the low molecular weight of cytochrome c allows for passage of cytochrome $\mathrm{c}$ from the microdialysis membrane into the interstitial fluid, where it may be reduced outside of the probe. Additionally, the high sample volume requirement necessitates a need for either a very high perfusion flow rate, ultimately limiting the potential for diffusion; or a need for long duration collection times, which places a greater time constraint on the study protocol design.
Importantly, the specificity of cytochrome c has been questioned (Münzel et al. 2002, Murrant and Reid 2001, Thomson et al. 1995). Cytochrome c has been found to be reduced by the hydroxyl radical or nitric oxide (Murrant and Reid 2001) and can be oxidized by ONOO(Thomson et al. 1995). Since high levels of $\mathrm{O}_{2}{ }^{--}$are associated with low nitric oxide bioavailability and potentially with elevated $\mathrm{ONOO}^{-}$production, the cytochrome c reduction assay may not be appropriate for comparing ROS production levels in a pathological state relative to a control group. In addition, it has recently been suggested that cytochrome $\mathrm{c}$ reduction is appropriate for quantifying $\mathrm{O}_{2}{ }^{-}$released during the respiratory burst of neutrophils or by isolated enzymes, but the assay is likely not sensitive enough to detect small quantities of $\mathrm{O}_{2}{ }^{--}$produced by nonphagocytic cells (Dikalov and Harrison 2014). As HRP is too large to pass out of the membrane, the methods utilized in the present study strictly detect ROS that diffuse into the microdialysis membrane with high specificity to $\mathrm{H}_{2} \mathrm{O}_{2}$, and can be measured quickly and easily in non-contracting skeletal muscle of healthy individuals that likely produce levels of ROS that are on the low end of what is to be expected in a human population.

The technique described in the present study has a wide range of utility, as it can be used safely in humans with minimally invasive techniques. This technique could be used to measure ROS in pathological states, and could be used to test pharmacological efficacy of oral antioxidant therapies or drugs targeted to improvement of components of the metabolic syndrome. An additional advantage to the microdialysis technique is that it can be utilized with pharmacologic agents or enzyme targeted antioxidants delivered directly to the muscle via microdialysis to test the localized effect of these agents on extracellular ROS levels. Furthermore, these measures could be performed with simultaneous monitoring of blood flow (Hickner et al. 1992) and metabolism (Hershberger et al. 2004) via microdialysis.

\section{Conflict of Interest}

There is no conflict of interest.

\section{Acknowledgements}

This research was supported in part by grants from the American College of Sports Medicine Foundation (J.D.L.) and the National Institutes of Health 1R15HL113854-01A1 (R.C.H.). 


\section{References}

CLOSE GL, KAYANI AC, ASHTON T, MCARDLE A, JACKSON MJ: Release of superoxide from skeletal muscle of adult and old mice: an experimental test of the reductive hotspot hypothesis. Aging Cell 6: 189-195, 2007.

DIKALOV S: Cross talk between mitochondria and NADPH oxidase. Free Radic Biol Med 51: 1289-1301, 2011.

DIKALOV SI, HARRISON DG: Methods for detection of mitochondrial and cellular reactive oxygen species. Antioxid Redox Signal 20: 372-382, 2014.

FISHER-WELLMAN KH, NEUFER PD: Linking mitochondrial bioenergetics to insulin resistance via redox biology. Trends Endocrinol Metab 23: 142-153, 2012.

HELLSTEN Y, NIELSEN JJ, LYKKESFELDT J, BRUHN M, SILVEIRA L, PILEGAARD H, BANGSBO J: Antioxidant supplementation enhances the exercise-induced increase in mitochondrial uncoupling protein 3 and endothelial nitric oxide synthase mRNA content in human skeletal muscle. Free Radic Biol Med 43: 353$361,2007$.

HERSHBERGER AM, MCCAMMON MR, GARRY JP, MAHAR MT, HICKNER RC: Responses of lipolysis and salivary cortisol to food intake and physical activity in lean and obese children. $J$ Clin Endocrinol Metab 89: 4701-4707, 2004.

HICKNER RC, ROSDAHL H, BORG I, UNGERSTEDT U, JORFELDT L, HENRIKSSON J: The ethanol technique of monitoring local blood flow changes in rat skeletal muscle: implications for microdialysis. Acta Physiol Scand 146: 87-97, 1992.

HICKNER RC, BONE D, UNGERSTEDT U, JORFELDT L, HENRIKSSON J: Muscle blood flow during intermittent exercise: comparison of the microdialysis ethanol technique and ${ }^{133}$ Xe clearance. Clin Sci 86: 15-25, 1994.

JANERO DR: Malondialdehyde and thiobarbituric acid-reactivity as diagnostic indices of lipid peroxidation and peroxidative tissue injury. Free Radic Biol Med 9: 515-540, 1990.

MCARDLE A, PATTWELL D, VASILAKI A, GRIFFITHS RD, JACKSON MJ: Contractile activity-induced oxidative stress: cellular origin and adaptive responses. Am J Physiol 280: C621-C627, 2001.

MCARDLE A, VAN DER MEULEN J, CLOSE GL, PATTWELL D, VAN REMMEN H, HUANG TT, RICHARDSON AG, EPSTEIN CJ, FAULKNER JA, JACKSON MJ: Role of mitochondrial superoxide dismutase in contraction-induced generation of reactive oxygen species in skeletal muscle extracellular space. Am J Physiol 286: C1152-C1158, 2004.

MOHANTY JG, JAFFE JS, SCHULMAN ES, RAIBLE DG: A highly sensitive fluorescent micro-assay of $\mathrm{H}_{2} \mathrm{O}_{2}$ release from activated human leukocytes using a dihydroxyphenoxazine derivative. J Immunol Methods 202: 133-141, 1997.

MÜNZEL T, AFANAS'EV IB, KLESCHYOV AL, HARRISON DG: Detection of superoxide in vascular tissue. Arterioscler Thromb Vasc Biol 22: 1761-1768, 2002.

MURRANT CL, REID MB: Detection of reactive oxygen and reactive nitrogen species in skeletal muscle. Microsc Res Tech 55: 236-248, 2001.

PATTWELL D, MCARDLE A, GRIFFITHS RD, JACKSON MJ: Measurement of free radical production by in vivo microdialysis during ischemia/reperfusion injury to skeletal muscle. Free Radic Biol Med 30: 979-985, 2001.

POLI G, LEONARDUZZI G, BIASI F, CHIARPOTTO E: Oxidative stress and cell signalling. Curr Med Chem 11: 1163-1182, 2004.

THOMSON L, TRUJILLO M, TELLERI R, RADI R: Kinetics of cytochrome c2+ oxidation by peroxynitrite: implications for superoxide measurements in nitric oxide-producing biological systems. Arch Biochem Biophys 319: 491-497, 1995.

VOTYAKOVA TV, REYNOLDS IJ: Detection of hydrogen peroxide with Amplex Red: interference by NADH and reduced glutathione auto-oxidation. Arch Biochem Biophys 431: 138-144, 2004.

WESELER AR, BAST A: Oxidative stress and vascular function: implications for pharmacologic treatments. Curr Hypertens Rep 12: 154-161, 2010.

ZHANG Q, PISTON DW, GOODMAN RH: Regulation of corepressor function by nuclear NADH. Science 295: 18951897, 2002. 
ZHOU M, DIWU Z, PANCHUK-VOLOSHINA N, HAUGLAND RP: A stable nonfluorescent derivative of resorufin for the fluorometric determination of trace hydrogen peroxide: applications in detecting the activity of phagocyte NADPH oxidase and other oxidases. Anal Biochem 253: 162-168, 1997. 